\title{
Arterial Cerebral Blood Volume-Weighted Functional MRI Using Pseudocontinuous Arterial Spin Tagging (AVAST)
}

\author{
Hesamoddin Jahanian, ${ }^{1 *}$ Scott Peltier, ${ }^{2}$ Douglas C. Noll, ${ }^{2}$ and Luis Hernandez Garcia ${ }^{2}$
}

Purpose: Neurovascular regulation, including responses to neural activation that give rise to the blood oxygenation leveldependent (BOLD) effect, occurs mainly at the arterial and arteriolar level. The purpose of this study is to develop a framework for fast imaging of arterial cerebral blood volume (aCBV) signal suitable for functional imaging studies.

Methods: A variant of the pseudocontinuous arterial spin tagging technique was developed in order to achieve a contrast that depends on aCBV with little contamination from perfusion signal by taking advantage of the kinetics of the tag through the vasculature. This technique tailors the tagging duration and repetition time for each subject. The proposed technique, called AVAST, is compared empirically with BOLD imaging and standard (perfusion-weighted) arterial spin labeling (ASL) technique, in a motor-visual activation paradigm.

Results: The average Z-scores in the activated area obtained over all the subjects were 4.25, 5.52, and 7.87 for standard ASL, AVAST, and BOLD techniques, respectively. The aCBV contrast obtained from AVAST provided $80 \%$ higher average signal-to-noise ratio and $95 \%$ higher average contrast-to-noise ratio compared with that of the standard ASL measurements.

Conclusion: AVAST exhibits improved activation detection sensitivity and temporal resolution over the standard ASL technique, in functional MRI experiments, while preserving its quantitative nature and statistical advantages. AVAST particularly could be useful in clinical studies of pathological conditions, longitudinal studies of cognitive function, and studies requiring sustained periods of the condition. Magn Reson Med 73:1053-1064, 2015. ㄷ 2014 Wiley Periodicals, Inc.

Key words: arterial cerebral blood volume; fMRI; arterial spin labeling; pCASL

\section{INTRODUCTION}

Cerebral blood volume (CBV) is a very important measurement for the study of a number of neurological disorders that involve vascular dysregulation. These include hypertension, stroke (1,2), small brain infarcts (3),

\footnotetext{
${ }^{1}$ Department of Radiology, Stanford University, Stanford, California, USA ${ }^{2}$ Functional MRI Laboratory, University of Michigan, Ann Arbor, Michigan, USA.

Grant sponsor: National Institute of Health; Grant numbers: EB004346 and DA026077.

*Correspondence to: Hesamoddin Jahanian, Ph.D., Lucas Center for Imaging, 1201 Welch Road, Mailcode 5488, Stanford University, Stanford, CA 94305-5488. E-mail: hesamj@stanford.edu

Correction added after online publication 30 April 2014. Line 1 of Table 1 was corrected to "CNR in activated area." Line 2 of Table 1 was corrected to "SNR in whole brain."

Received 16 September 2013; revised 14 February 2014; accepted 25 February 2014

DOI 10.1002/mrm.25220

Published online 18 April 2014 in Wiley Online Library (wileyonlinelibrary. com).

(c) 2014 Wiley Periodicals, Inc.
}

Alzheimer disease (4), astrocytoma and other brain tumors $(5,6)$, and cerebral stenoses (7). The ability to separate the arterial compartment from the venous compartment of CBV may provide additional information not obtainable by imaging the total CBV [e.g., in stenoocclusive disease of the internal carotid artery (8)]. It may also provide insights into vascular control mechanisms in both healthy and diseased tissue $(6,8,9)$.

Cerebral perfusion is regulated at the arteriolar level. Thus, measurement of arterial CBV (aCBV) can be an essential indicator of vascular reactivity (9) and thus can also be used to study the clinical conditions during which the normal relation between cerebral blood flow (CBF), CBV, and blood oxygenation is disrupted. Arterial and venous vessels show considerably different responses during neuronal activation $(10,11)$, and thus imaging of arterial cerebral blood volume (aCBV) can also provide important information for the study of human brain function and cognition. Indeed, functional magnetic resonance imaging (fMRI) using physiological parameters such as CBF or CBV, unlike BOLD fMRI, provides a quantifiable contrast and is also more closely related to neural activity (12-14). These methods can also be much less sensitive to the local susceptibility artifacts due to the use of shorter echo times (TEs) or by employing imaging techniques that are less sensitive to the field inhomogeneity (e.g., fast spin echo). This would have specific use in areas of the brain that lie near air spaces such as frontal and low brain regions, which is an issue plaguing the BOLD effect (15). Furthermore, dynamic measurement of aCBV can provide critical information about the mechanism of the blood oxygen level-dependent (BOLD) contrast itself (16).

Because of these potential advantages, CBF-, CBV-, and aCBV-based functional MRI methods have recently received attention as alternative and complementary techniques to BOLD fMRI. These methods, however, generally have been hampered in terms of signal-to-noise ratio (SNR), temporal resolution, and multislice imaging capability.

Different methods have been proposed for noninvasive measurement of aCBV in animals (17) and humans (1821). Most prominently, iVASO (21) uses the difference in $\mathrm{T} 1$ relaxation time of blood and tissue to assess the aCBV. This method, however, is based on a set of assumptions about the arterial arrival time and T1 values, which requires further verification (22). Alternatively, aCBV can also be estimated from the signal obtained from variants of arterial spin labeling (ASL) techniques. Some techniques obtain ASL images at different postlabeling delays and quantify the aCBV by modeling the collected signal (18-20). Several other techniques take differencing ASL signals with and without 
flow-crusher gradients in order to isolate the arterial signal (18,22-24). Another technique, called MOTIVE (17), uses magnetization transfer along with ASL to modulate the tissue and blood signals and estimate the aCBV in animals. Although recent improvements in imaging and labeling techniques such as three-dimensional (3D) GRASE readout (25), background suppression (26), and optimized labeling schemes (27-30) can reduce practical issues of these techniques, and despite the fact that all of these methods have been shown to be useful in various applications, many are still not practical for clinical studies or cognitive psychology experiments. These experiments are often more demanding in terms of spatial coverage and resolution as well as temporal resolution, and hence may not permit long acquisition times or additional measurements of physiological constants.

The purpose of this study is to develop a simple, novel, dynamic aCBV measurement technique based on pseudocontinuous arterial spin labeling (pCASL) $(30,31)$ that is suitable for functional imaging experiments. We refer to our proposed measurement technique as arterial volume using arterial spin tagging (AVAST). AVAST tailors the tagging duration and repetition time (TR) for each subject to achieve a contrast that depends on aCBV with little or no contamination from perfusion signal. AVAST also allows for a fast acquisition rate (TR $\sim 2.2$ s), making it suitable for dynamic fMRI experiments.

AVAST offers sensitivity to brain activation that is on par with BOLD imaging and is superior to CBF ASL, while maintaining many advantages of CBF ASL imaging such as reduced sensitivity to susceptibility artifact, improved localization (12), quantifiable signal, and reduced sensitivity to the MR scanner signal drift (32). We demonstrate AVAST using numerical simulations and in vivo experimental data. We then compare it with standard (CBF) ASL and BOLD techniques in terms of sensitivity, reproducibility, locus of activation, and temporal response for fMRI experiments.

\section{METHODS}

\section{Theory}

Our strategy was to design the timing parameters of a continuous ASL sequence such that the signal was acquired when the tagged spins were primarily still in the arteries before they had filled the capillaries. Through the adjustment of the timing parameters, the contribution from tagged spins in the capillary and tissue compartments was the same in both the control and tagged images and therefore could be subtracted out. We considered a given voxel to consist of three separate compartments through which blood water flows: the arterial compartment, the capillary and tissue compartment, and the venous compartment. Here we simply refer to the combined capillary and extravascular tissue as "tissue." In an ASL experiment, the tagged spins entered the voxel via the arterial compartment and exchanged through the capillaries/tissue on its way to the venous compartment, from which it left the voxel. At 3T and below, T1 decay prevented the tagged spins from retaining their tag by the time they reached the venous compartment, so it could be safely neglected.
Therefore, for our purposes, the observed ASL signal can be split into an arterial and a tissue compartment $\left(S_{A S L}=S_{a}+S_{t}\right)$ and modeled separately. Arterial signal $\left(S_{a}\right)$ in a continuous ASL experiment can be expressed by adapting the general kinetic model (33) for magnetization difference between tagged and control images as follows:

$$
\begin{gathered}
S_{a}(t)=\Delta M_{a}(t)=2 \alpha \cdot M_{a 0} \cdot a C B V \cdot c(t) \\
c(t)=\operatorname{rect}(t, w) *\left[k\left(t-\tau_{1}\right) \cdot m(t)\right] \\
k(t)=\left[\frac{S^{(1+S P)}}{\Gamma(1+\mathrm{SP})} t^{S P} e^{-S t}\right] \\
m(t)=e^{-t / T_{1 a}}
\end{gathered}
$$

where $M_{a 0}$ is the equilibrium magnetization of arterial blood and $M_{a}(t)$ describes the magnetization of arterial blood at time $t$. $a C B V$ is the aCBV fraction in a given voxel, $\alpha$ is the inversion efficiency of the ASL pulse, and $\operatorname{rect}(t, w)$ describes a rectangular tagging function of duration $\mathrm{w}$. The mean time it takes for the tagged spins to reach the voxel is $\tau_{1}$, or the "arterial arrival time." The bolus of tagged spins experiences longitudinal relaxation, as well as dispersion of velocities as it moves up the arterial network. $m(t)$ represents the longitudinal magnetization relaxation function defined as the fraction of original longitudinal magnetization tag carried by the water molecules that remains at a time $t$ after their arrival in the voxel and is characterized by a decaying exponential with time constant $T_{1 a}$ ( $T_{1}$ decay of blood in artery). The dispersion of the tag during travel to the imaging plane can be approximated by convolving the input function with a Gamma kernel $k(t)(34,35)$ where S and P characterize the "sharpness" and the "time-topeak" of the kernel.

The arterial tag in turn serves as an input to the tissue compartment. The tissue signal $\left(S_{t}\right)$, can be expressed as a second convolution

$$
S_{t}(t)=S_{a}(t) \cdot f *\left\{e^{-\frac{f}{\lambda}\left(t-\tau_{2}\right)} \cdot e^{-\left(t-\tau_{2}\right) T_{1 t}}\right\} .
$$

Here, the second term in the convolution captures the fraction, $\mathrm{f}$ (perfusion), of tag from the tissue after a short delay or "tissue transit time," $\tau_{2}$, and the decay of the tag's longitudinal magnetization in the tissue compartment with time constant $T_{1 t}\left(T_{1}\right.$ decay of blood in tissue). $\lambda$ is the blood-brain partition coefficient for water in the whole brain (36).

Intuitively, this framework describes the process by which the tag is taken up and washes out of the arterial compartment and then the tissue compartment of a given voxel. The goal of the following simulations was to find a range of pulse sequence timing parameters that produces an ASL signal that is primarily dominated by the arterial compartment and to characterize the observed signals.

\section{Simulations}

We simulated the general kinetics of the ASL signal at different TRs and tagging durations using Eqs. [1-5] in order to explore the effect of the pulse sequence timing 

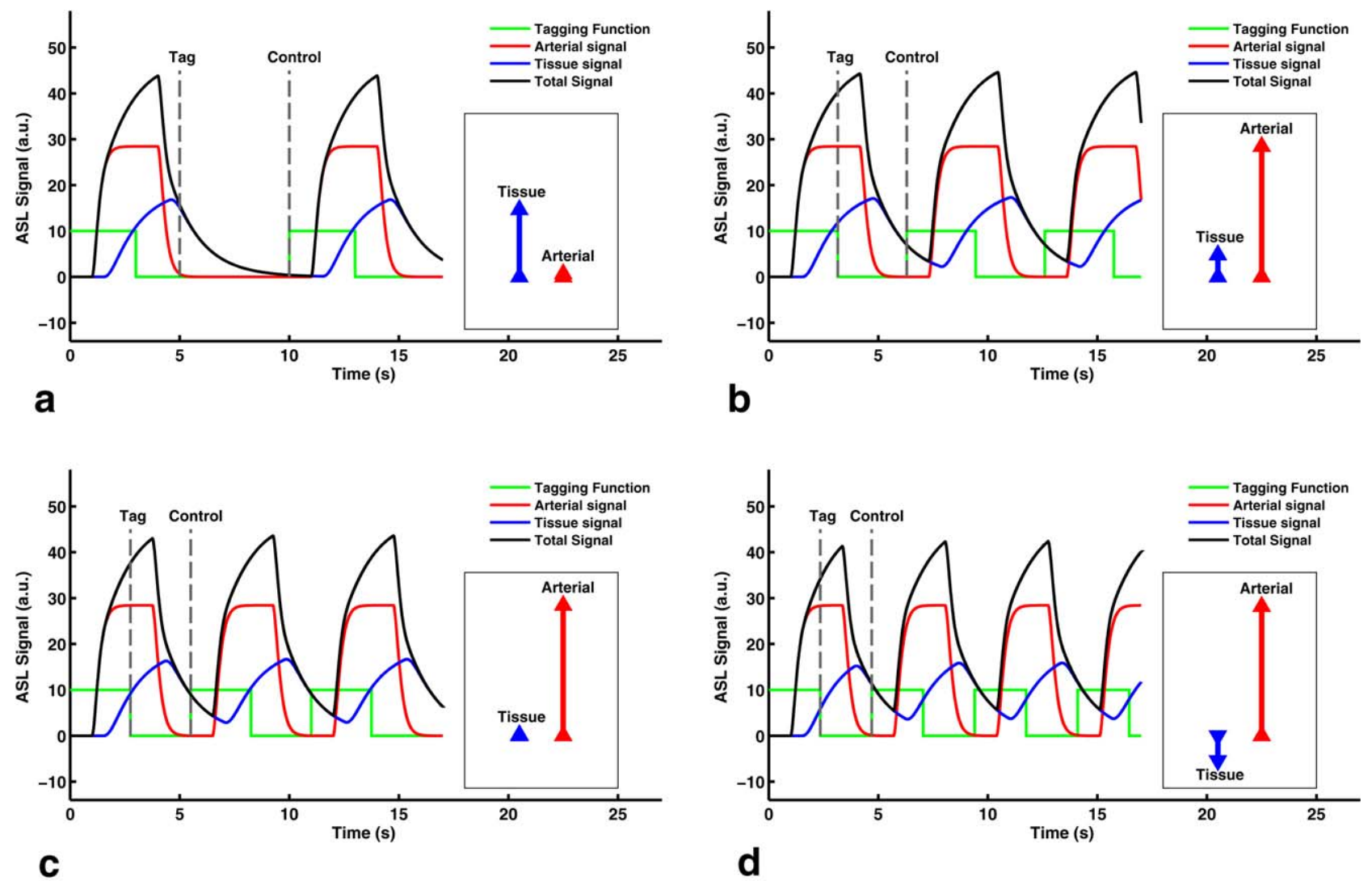

FIG. 1. Simulated kinetics of the concentration of label in the tissue arising from arterial and tissue compartments in a given voxel using different timing parameters. The tagging function, arterial and tissue signals, and total ASL signal time courses are shown using green, red, blue, and black curves, respectively. The timing of the acquisition of the first pair of control and tagged images is shown using dotted vertical lines. The individual contributions of tissue and arterial compartments in the subtraction of tagged from control image are also indicated on the right side of each curve by blue and red arrows, respectively. a: Standard continuous ASL experiment employing a long postinversion delay (large tissue signal, no arterial signal) yielding perfusion-weighted images. b: Continuous ASL experiment without postinversion delay (small tissue signal, large arterial signal. c: Continuous ASL experiment without postinversion delay and with optimal tagging duration for aCBV measurement (no tissue signal, large arterial signal). d: Continuous ASL experiment without postinversion delay and with shorter than optimal tagging duration for aCBV measurement (small negative tissue signal, large arterial signal).

parameters with respect to multiple arterial arrival and tissue transit times. We assumed that image acquisition happens instantly after the tagging period (i.e., no postinversion delays). We used the following constants in the model $\left[f=0.015 \mathrm{~mL} / \mathrm{g} / \mathrm{s}, M_{0 a}=3000, a C B V=2 \mathrm{~mL} /\right.$ $100 \mathrm{~mL}, T_{1 \mathrm{a}}=1.6 \mathrm{~s}(37), T_{1 \mathrm{t}}=1.4 \mathrm{~s}$ (38), $\alpha=0.8$ (28), $\lambda=$ $0.9 \mathrm{~mL} / \mathrm{g}] . M_{0 \mathrm{a}}$ is an arbitrary signal intensity representing the equilibrium magnetization of arterial blood. To account for the dispersion of tagged blood, we convolved the input function with input function with the Gamma kernel presented in Eq. [3] using the parameters suggested by Chappell et al. (35) ( $\mathrm{s}=0.38, P=0.11$ ). Arterial arrival time $\left(\tau_{1}\right)$ and tissue transit time $\left(\tau_{2}\right)$ were $1 \mathrm{~s}$ and $0.5 \mathrm{~s}(39)$.

Figure 1 shows a simulation of the ASL signal arising from the arterial and tissue compartments separately (recall that the ASL signal consists of the subtraction of a tagged image from a control image) acquired with several different timing parameters. The contribution of each compartment to the ASL signal depends on the pulse sequence's timing parameters along with each subject's arterial arrival time, $\tau_{1}$, and local arterial delay or tissue transit time, $\tau_{2}$. If the tagging duration is relatively long (>1 s) and a delay greater than the arterial arrival time is introduced between the tagging and acquisition portions of the sequence, the tag clears the arterial compartment and fills the tissue compartment. Thus, the arterial contribution to the ASL signal could be eliminated using a long postinversion delay (Fig. 1a) $(40,41)$. Without a postinversion delay (Fig. 1b), the ASL signal was a mixture of arterial and tissue signals. Note that changing the tagging duration and the TR determined the amount of tag in the arterial and tissue compartments when the control and tagged images were collected (Fig. 1b-1d). As can be seen in (Fig. 1c), by choosing the proper TR and tagging duration, it was possible to have the same contribution from tissue in the control and tagged images. In this case, the tissue signal subtracted out in the ASL signal, and the remaining observed signal arose from the arterial compartment alone, which was our goal for this technique.

We used this framework to compute the contributions of the arterial and tissue compartments to the ASL signal as a function of tagging duration. Thus, we were able to identify a range of tagging durations for which the net signal was mostly arterial with small tissue contribution to the signal. 


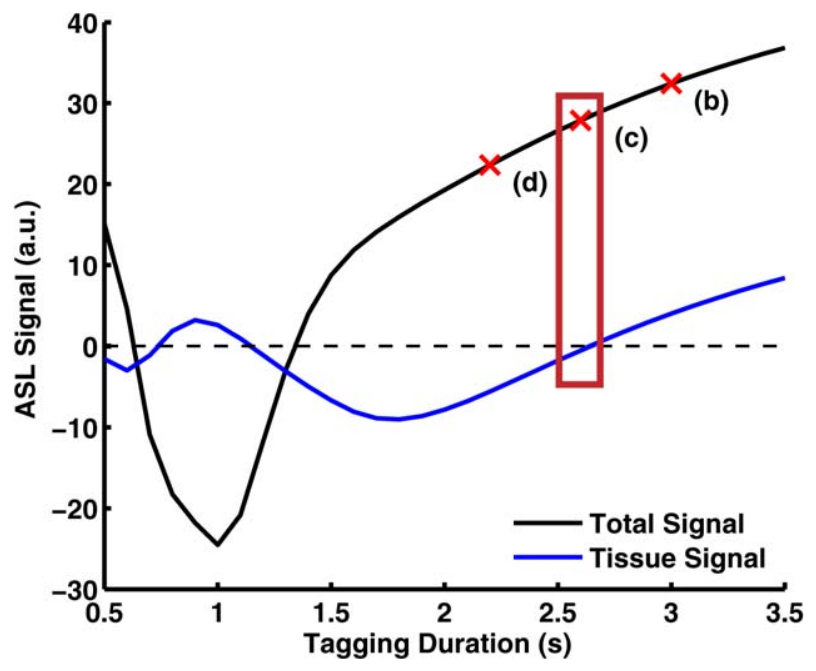

FIG. 2. Simulated ASL signal (control minus tagged images) as a function of tagging duration considering no postinversion delay. Arterial arrival time assumed to be $1 \mathrm{~s}$. TR was adjusted dynamically to accommodate the increasing tagging duration. Points highlighted with red x's correspond to the kinetic curves shown in Fig. 1b, 1c, and $1 \mathrm{~d}$.

The contribution of the arterial and tissue compartments to the ASL signal as a function of tagging duration is shown in Fig. 2, assuming that the image acquisition happened at the end of the tagging period. The highlighted points (red crosses) on the black curve in Fig. 2 correspond to the timing parameters shown in Fig. 1b$1 \mathrm{~d}$. As can be seen in Fig. 2, there are several tagging durations for which the tissue contribution to the arterial signal is zero. At these "zero-crossings" of the tissue contribution curve (blue), there is still $\sim 50 \%-75 \%$ of arterial contribution to the ASL signal. Among these points, the location indicated in Fig. 2 by a red rectangle suggests a very stable range of tagging times for which the net signal is mostly arterial (i.e., the tissue contribution to the signal is $<5 \%$ ). Consequently, using this tagging duration, the fractional ASL signal change upon activation during a functional study is related to the local changes in the arterial blood volume. We refer to this timing combination as the "aCBV point" and use it to collect aCBV-weighted images.

In practical terms, the first task in the AVAST technique is to identify the timing parameters of the aCBV point by testing a range of tagging durations with flow suppression gradients, in order to isolate the contribution of the tissue signal. Once the aCBV point is identified in a preliminary scan, a time series of dynamic aCBV images can be collected by fixing the pulse sequence parameters to those of the aCBV point and turning off the flow suppression gradients.

\section{Sensitivity to Timing Variations}

The location of the aCBV point is determined by the subject's specific arterial arrival and tissue transit times relative to the image acquisition parameters. Therefore, the best selection of parameters will vary from subject to subject and over brain regions. To evaluate the sensitivity of AVAST to these timing variations, we conducted a simulation study. We simulated the ASL signal for a case in which the aCBV sequence was calibrated for a specific region with a given set of physiological parameters [arterial arrival time of $1 \mathrm{~s}$ and tissue transit time of $0.5 \mathrm{~s}$ (39)]. Keeping the acquisition parameters the same, in Fig. 3a and $3 \mathrm{~b}$, we calculated what happens to the signals when the voxels' arterial arrival and tissue transit times differed up to $0.5 \mathrm{~s}$ from the original calibration in order to illustrate the amount of tissue contamination that can be potentially found using this method. As shown in Fig. 3a

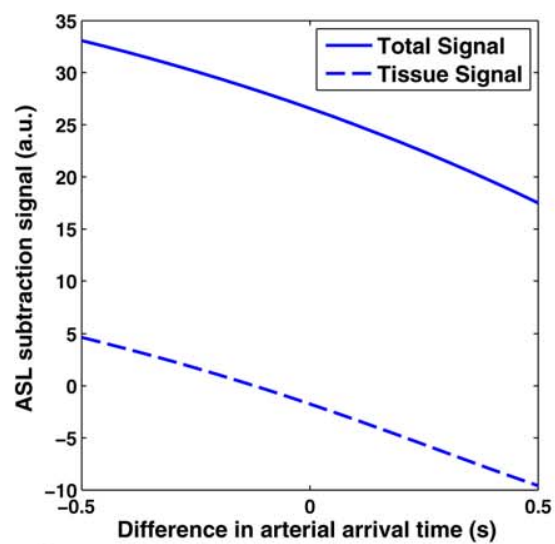

a
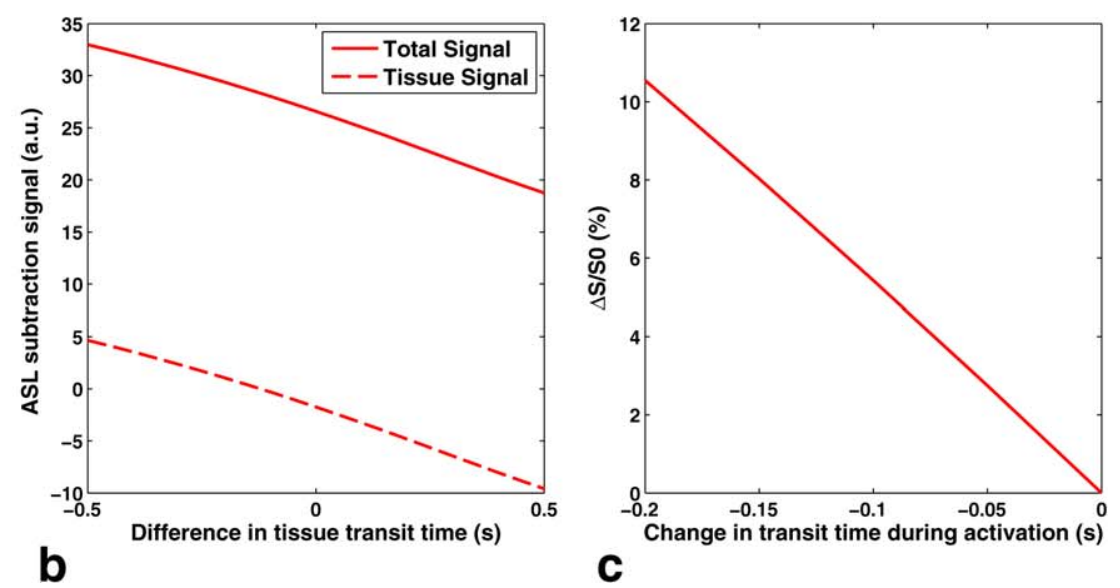

FIG. 3. Simulation study of the sensitivity of AVAST to the arterial arrival time and tissue transit time variability across different areas of the brain or under different conditions (e.g., activation). aCBV acquisition was optimized for a voxel where the arterial arrival time $\left(\tau_{1}\right)$ and the tissue transit time $\left(\tau_{2}\right)$ are 1 and $0.5(\mathrm{~s})$, respectively. The individual contributions of arterial and tissue compartments in the ASL subtraction signal (control minus tagged images) were calculated for a broad range of transit times, different ( $\pm 0.5 \mathrm{~s})$ from the ones used in the aCBV optimization. Plots show total (arterial + tissue) and tissue signals. Subplot (a) indicates the effect of variation in arterial arrival time. Subplot (b) indicates the effect of variation in tissue transit time. Simulated change in the AVAST signal between rest and activation resulting from the reduction in the tissue transit time in response to neural activation is also shown in subplot (c). 
and $3 \mathrm{~b}$, if we were wrong by $50 \%$ in the arterial arrival time or tissue transit time, the contribution from the tissue-tagged spins to the aCBV signal was $\sim 10 \%$.

In addition to spatial variability, tissue transit time also changes during the neuronal activation. It has been shown that neuronal activation is accompanied by a local reduction in tissue transit time of $\sim 0.150 \pm 0.045 \mathrm{~s}$ (42). As a result of this reduction, there will be a change in the total signal between rest and activation conditions. Figure 3c shows the relative change in the total signal between rest and activation as a function of the reduction in the tissue transit time during neuronal activation. Since the vasodilation happens locally at the area of activation, in this simulation we assumed that the neuronal activation mainly affects the tissue transit time and not the arterial arrival time. It is important to note that this change is due to the shift in the aCBV point and not due to the actual change in aCBV due to activation. It can be seen that there could be as much as a $\sim 10 \%$ increase in the signal because of the activationinduced reduction in the transit time.

\section{Quantification}

Using Eqs. [1-4], it is possible to quantify the aCBV signal obtained using AVAST. To this end, it is necessary to estimate the arterial arrival time from the tagging plane at the neck to the imaging plane. We estimated the arterial arrival time by varying the tagging duration and TR and following the changes in the ASL signal without using flow crusher gradients $(42,43)$. The signal can then be quantified using the following equations:

$$
\begin{gathered}
a C B V=\frac{\Delta M(T R)}{2 \alpha \cdot M_{a 0} \cdot C(T R)} \\
M_{a 0}=\frac{M_{a}(T R)}{\left(\sin \theta \cdot e^{-T R / T_{1 a}}\right)}
\end{gathered}
$$

in which $\theta$ is the flip angle of $3 \mathrm{D}$ imaging pulse and $M_{a}$ is the equilibrium magnetization of the arterial blood in the sagittal sinus measured from the mean of the control images.

\section{In Vivo Experiments}

\section{D Image Acquisition}

We employed a 3D imaging pulse sequence with multishot (segmented) readouts for imaging the aCBV signal. The readout is a 3D stack of spiral trajectories implemented using radiofrequency spoiling . Besides a modest increase in SNR relative to two-dimensional acquisition (44), the $3 \mathrm{D}$ acquisition ensures the same acquisition delay for the entire imaging volume relative to the tagging period and therefore provides us with the capability to measure the aCBV in a volume rather than a single slice (44). If we were to use sequential two-dimensional imaging, each slice would be acquired at a different postinversion delay and consequently, the optimality of the aCBV acquisition would be slice-dependent.

The 3D gradient echo spiral images in this study were obtained using the following parameters: pulse $\mathrm{TE}=$ $0.004 \mathrm{~s}$; field of view $=24 \mathrm{~cm}$; slab thickness $=11 \mathrm{~cm}$; and matrix size $=64 \times 64 \times 22$. Planes along the $\mathrm{k}_{\mathrm{z}^{-}}$ dimension were sampled starting at the center of $k$-space and moving outward alternating between negative and positive $\mathrm{k}_{\mathrm{z}}$ increments using 22 excitations, each taking $0.023 \mathrm{~s}$. We used a cubic flip-angle schedule from 15 to 90 degrees as we traversed k-space along the slab-select direction, in order to reduce blurring along the sliceencoding dimension (44). Due to use of a low flip angle we did not consider spin history effects and assumed those to be negligible. The TR and tagging durations used for each set of experiments are explained in the subsequent sections.

\section{aCBV calibration}

Our simulations suggest that the timing parameters producing the aCBV images is determined by each subject's specific arterial arrival and tissue transit times relative to the image acquisition parameters. Therefore, the best selection of parameters varied from subject to subject, although our simulations indicate that the technique is not very sensitive to errors in transit times. For each subject, we identified the timing parameters of the aCBV point by running a calibration sequence before the functional experiment, as follows. The calibration sequence tests the ASL signal over a range of TRs in the presence of flow suppression gradients (similar to the blue curve in Fig. 2). Arterial signals were suppressed by using a pair of bipolar crusher gradients $(b=4 \mathrm{~s} / \mathrm{mm} 2)$ along the Z-direction providing a cut off velocity of $1 \mathrm{~cm} / \mathrm{s}$. Guided by the simulation result and by previous experience $(42,43,45)$, TR was varied from 1.5 to $2.5 \mathrm{~s}$, steps of $0.1 \mathrm{~s}$, while keeping the tagging duration $0.5 \mathrm{~s}$ shorter than TR to allow the acquisition of 22 slices using for the calibration sequence. For each TR, we collected eight tag/control images and averaged them together during postprocessing. The total scan time for the aCBV calibration using these parameters was $352 \mathrm{~s}$.

The images were reconstructed, pairwise subtracted, and averaged for each TR. The TR and tagging duration yielding the lowest ASL signal in the presence of flow crusher gradients was selected as optimal for aCBV (i.e., aCBV point highlighted with a red box in Fig. 2).

\section{Functional Experiments}

Using the obtained aCBV parameters, we performed a functional aCBV experiment and compared it with the result of functional CBF and BOLD experiments. Ten subjects were scanned using a 3.0 T Signa Excite scanner (General Electric, Waukesha, Wisconsin, USA). We employed pseudocontinuous tagging pulses $(30,31)$ for functional $\mathrm{CBF}$ and aCBV experiments. The tagging efficiency of the pCASL pulse sequence was optimized by correcting for the field off-resonance (28). Image acquisition was performed employing the above-mentioned segmented 3D spiral acquisition pulse.

Subjects performed a simultaneous motor (sequential finger opposition) and visual (8-Hz flashing checkerboard) activation task while being scanned using BOLDweighted imaging, perfusion-weighted ASL, and AVAST, as described below. The activation paradigm consisted of 
five cycles of alternating rest (30 s) and activation (30 s). Total functional scan time for all methods was $300 \mathrm{~s}$.

The first activation map was obtained using the functional aCBV scheme of AVAST (i.e., TR/tagging duration obtained from the calibration scan, no postinversion delay). The second map was obtained using a functional CBF scheme employing a standard ASL technique (TR = $4 \mathrm{~s}$; tagging duration $=2 \mathrm{~s}$; postinversion delay $=1.5 \mathrm{~s}$ ). A BOLD fMRI study was also conducted (single-shot gradient echo reverse spiral pulse sequence (46): $\mathrm{TE}=0.03$ $\mathrm{s}$; TR $=2 \mathrm{~s}$; flip angle $=90^{\circ}$; same field of view; slice location and resolution as aCBV and CBF experiments) for comparison purposes. Four dummy scans were collected in the beginning of all functional scans to make sure that steady state was reached. To compare the reproducibility of each method, we repeated the functional experiments on the same day, using each method for all subjects. All datasets were reconstructed, surround subtracted, and analyzed by estimation of standard general linear model using the custom-written software FASL01 (http://fmri.research.umich.edu/resources/software/shared_code.php).

\section{SNR and CNR Calculations}

We characterized our functional experiment by a linear model as described by Mumford and Nichols (47) and Hernandez-Garcia et al. (48):

$$
y_{t}=\beta_{0}+\beta_{1} x_{1 t}+\varepsilon_{t}
$$

for each time point $t=1, \ldots, n$. The first regressor and its coefficient parameter, $\beta_{0}$, indicate the baseline signal for the BOLD experiment and baseline subtraction ASL signal for the aCBV and CBF experiments. The second regressor $x_{1 t}$ with its regression coefficient, $\beta_{1}$, describes the signal (BOLD or subtraction ASL signal) difference due to activation and thus the amplitude of $\beta_{1}$ is indicative of contrast of the signal between rest and activation. The square root of the estimated variance of the effects of interest $\left(\sigma_{\beta_{0}}\right.$ and $\sigma_{\beta_{1}}$ ) also corresponds to noise. Therefore, the temporal SNR and CNR of the functional experiment conducted using each method can be calculated using:

$$
S N R=\underset{v \in V_{b r n}}{\operatorname{Mean}}\left(\frac{\beta_{0 V}}{\sigma_{\beta_{0 v}}}\right)
$$

And

$$
C N R=\underset{v \in V_{\text {act }}}{\operatorname{Mean}}\left(\frac{\beta_{1 \mathrm{v}}}{\sigma_{\beta_{0 v}}}\right)
$$

in which $V_{b r n}$ and $V_{a c t}$, respectively, represent total brain voxels and activated brain voxels in each experiment.

\section{RESULTS}

\section{aCBV Calibration}

Figure 4 shows the ASL signal acquired at different TRs over a wide range of tagging durations (0.6-3 s) with and without the flow crusher gradients in a region of interest containing 50 voxels of gray matter around the motor

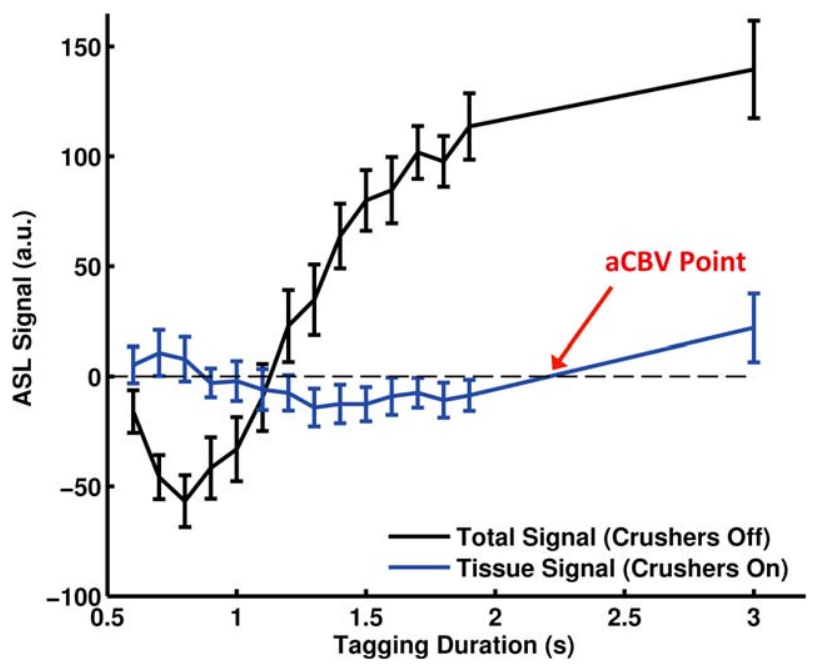

FIG. 4. Mean ASL signal measured for different tagging durations with (blue) and without (black) flow crusher gradients. For each measurement, TR was also adjusted to accommodate the increasing tagging duration. Error bars indicate standard deviation.

cortex area from one of the subjects. The black curve corresponds to the ASL signal without the flow crusher gradients and contains both arterial and tissue signals. The blue curve corresponds to the ASL signal with the flow crusher gradients, therefore it arises from the tissue signal (CBF). The tissue curve was used to estimate the optimal aCBV parameters by finding the aCBV point (pointed to by the red arrow). In agreement with our simulations (see Fig. 2), by incrementing the tagging duration and TR together, we were able to isolate the arterial contribution to the ASL signal. Note the temporal shift of the tissueonly ASL signals relative to the whole signals (arterial plus tissue). This is to be expected, since these spins must traverse the branching arteries and arterioles before they reach the capillary bed, where they can exchange. This delay corresponds to tissue transit time in our model. The range of TRs for the aCBV point estimated for our subjects was 2.0-2.5 s (mean/standard deviation $=2.26 / 1.8 \mathrm{~s}$ ). Also note that, in practice, only the tissue curve over a narrower range of tagging durations (1.5-2.5 s) is necessary to conduct an AVAST experiment.

\section{Functional Study Results}

All experiments produced activation in visual cortex, motor cortex and supplementary motor area using a Zthreshold of $5(Z>5)$. Figure 5 shows the activation maps from a representative subject obtained using standard ASL, AVAST, and BOLD fMRI overlaid on the corresponding average image. To help compare the activated areas structurally, they are also shown together on a separate image, with the overlapping areas highlighted in yellow. A 3D visualization of the activated areas is also presented. Active areas in the AVAST map were more focal (less scattered) than BOLD in all subjects. AVAST also produced wider activated areas compared with standard ASL. 


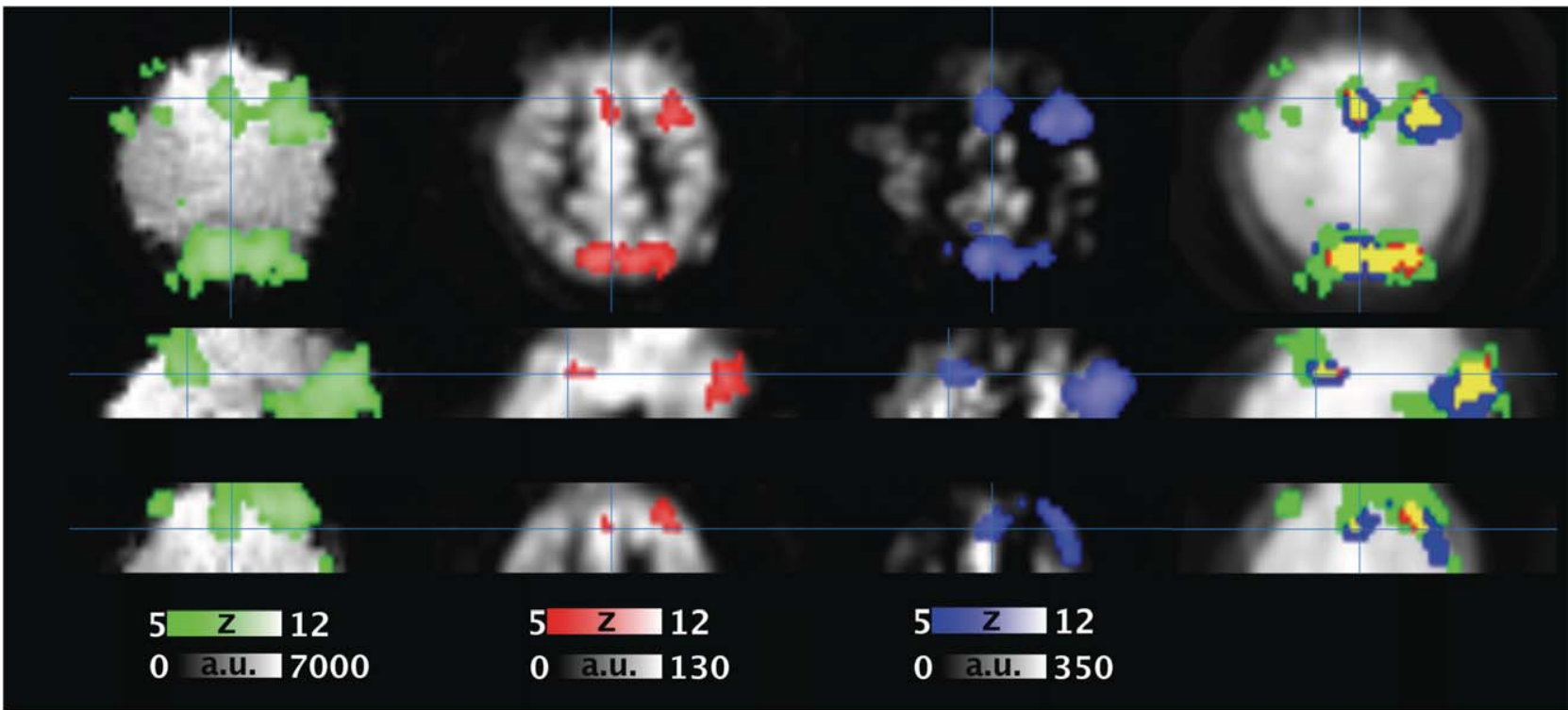

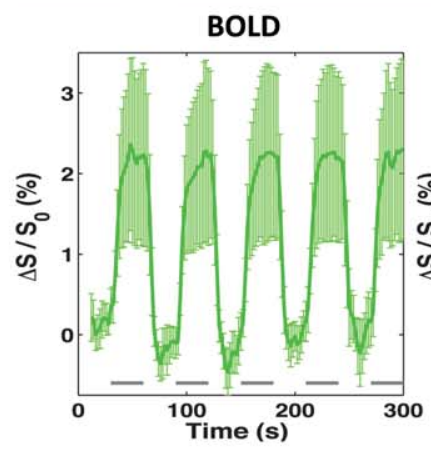

a

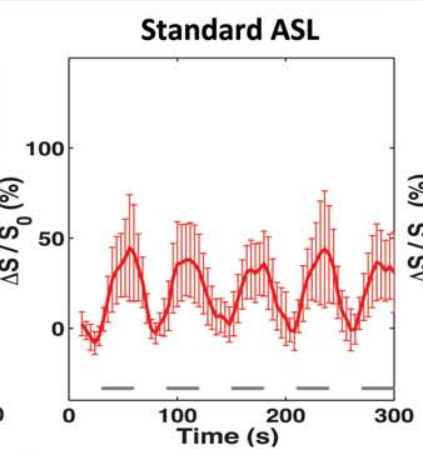

b

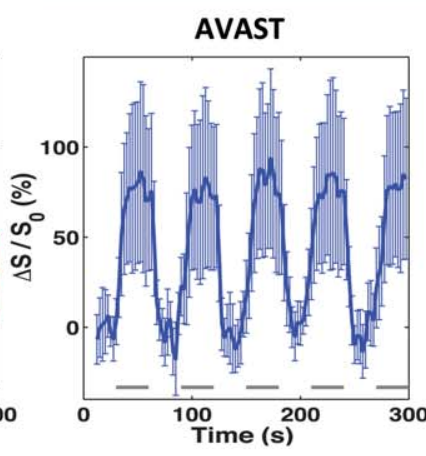

C

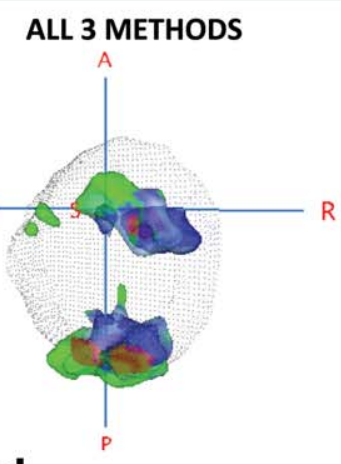

d

FIG. 5. Orthogonal slices illustrating activation maps $(Z>5)$ and their corresponding signal time course for a single representative subject obtained using (a) BOLD, (b) standard ASL (CBF contrast), and (c) AVAST (aCBV contrast) during visual and unilateral motor stimulation. Each activation map is overlaid on the average image corresponding to the technique used to generate that map. Time courses are averaged over the overlapping activated voxels for each technique. Error bars indicate the standard deviation across these voxels. Dark lines under the time courses show periods of stimulation. (d) The activated areas detected using each technique are shown in green (BOLD), red (standard ASL), and blue (AVAST). Overlapping voxels are shown in yellow. A 3D visualization of the activated areas also presented.

We compared the hemodynamic response of these methods by averaging the time course of the top $1 \%$ of voxels with the highest Z-score for each technique across the five cycles and subjects $(n=10)$. It is important to note that each method measures different physiological parameters and therefore the locus of the activation area detected using each method is different. Thus, to compare the CBF, aCBV, and BOLD time courses, it was not possible to choose an anatomical region of interest that fairly represented the activated area for all three methods. Moreover, since these methods have quite different sensitivities, comparing the results by choosing a fixed Z-threshold leads to a different number of voxels for comparison and will not be statistically equivalent for all methods. Therefore, we compared the most significant voxels of each method by choosing the voxels with the top $1 \%$ Z-scores for each method. Additionally, because the TR for AVAST experiments is not the same for different subjects, all aCBV time courses were first linearly interpolated to $\mathrm{TR}=2 \mathrm{~s}$ before averaging.

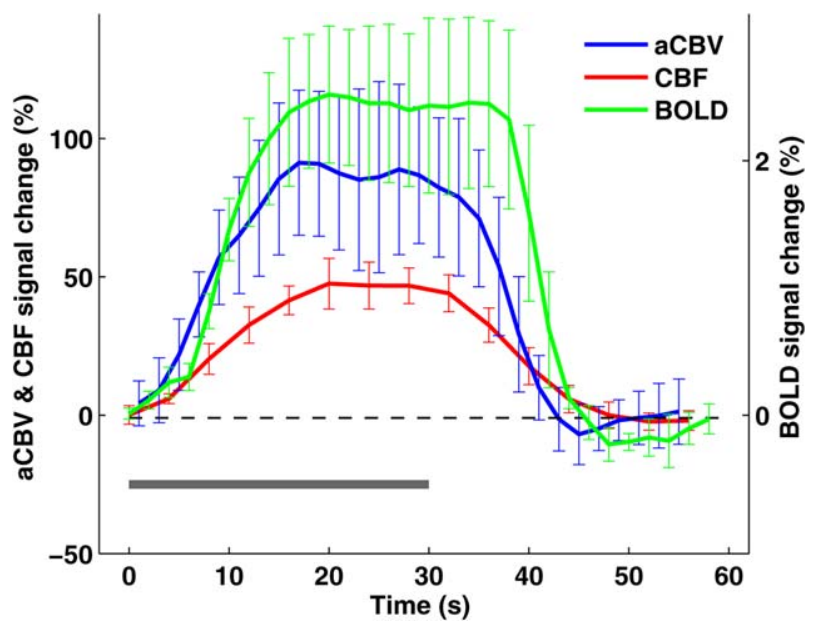

FIG. 6. Hemodynamic response averaged across five cycles of stimulation over all subjects $(n=10)$ for aCBV, CBF and BOLD $\mathrm{fMRI}$ techniques. Error bars indicate the standard deviation across subjects. Dark lines show the period of stimulation. 
Top $1 \%$ of Voxels

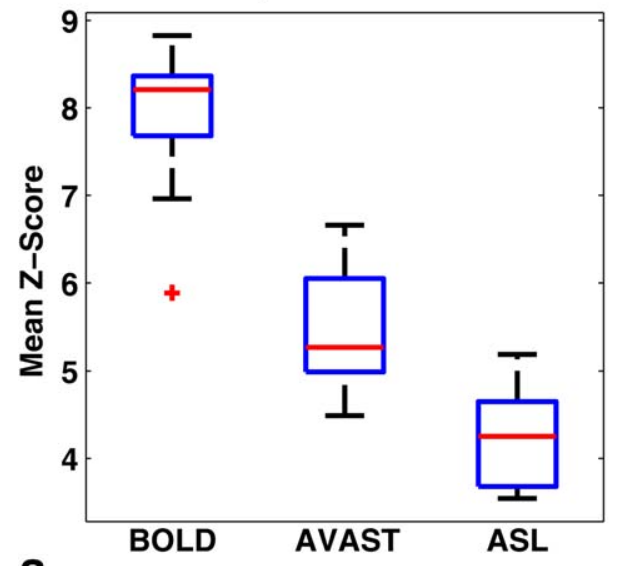

Overlapping Activated Voxels (Z>2)

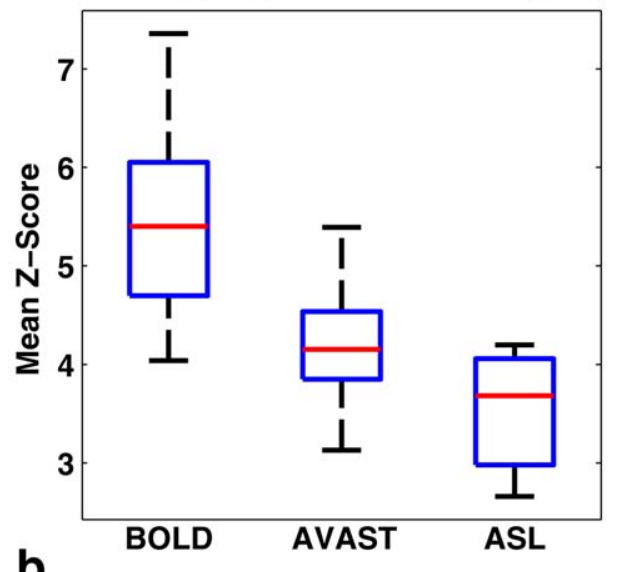

FIG. 7. Boxplots of mean Zscore values averaged over (a) the top $1 \%$ of voxels and (b) overlapping activated voxels $(Z>2)$ for BOLD, AVAST, and standard ASL techniques across all subjects.
Figure 6 shows the comparison of the hemodynamic response obtained using BOLD, standard ASL (CBFweighted contrast), and AVAST (aCBV-weighted contrast) methods. It can be seen that the CBF and BOLD responses have a delayed time to peak and slower return to baseline than aCBV. Poststimuli undershoot in the aCBV time courses was observed, but it returned to baseline faster than the BOLD signal. The average percentage signal changes during activation for BOLD, aCBV, and CBF signals were $2.4 \%, 85 \%$, and $46 \%$, respectively.

To compare the sensitivity of each method, we used two approaches: 1) we compared the mean of the top 1\% Z-scores (Fig. 7a); 2) we compared the mean Zscore within the overlapping activated areas defined as the voxels with $Z>2$ for all three methods (Fig. 7b). Figure 7 shows the calculated mean Z-scores for all 10 subjects. Average Z-scores calculated using the first approach for all the subjects were $4.25,5.52$, and 7.87 for standard ASL, AVAST, and BOLD, respectively. These numbers were $3.54,4.21$, and 5.42 using the second approach. These results indicate the superior activation detection sensitivity of AVAST compared with standard ASL.

In order to understand the spatial relationship between activation maps obtained with these methods in terms of locus of activation, we examined the overlap of the top $1 \%$ of the activated voxels between AVAST-BOLD, ASLAVAST, and ASL-BOLD. Figure 8a shows the calculated number of overlapping voxels for all 10 subjects. The average overlaps over all subjects were $54 \%, 36 \%$, and $46 \%$, respectively, which was expected considering the different physiological origin of each signal. In order to compare the reproducibility of each technique, we also calculated the overlap of the top $1 \%$ of the activated voxels between two runs for each method (Fig. 8b). The results indicate that AVAST has superior reproducibility compared with standard ASL but is still outperformed by BOLD.

The CNR and SNR of each method were calculated using Eqs. [9] and [10] and were averaged over all subjects (Table 1). It is important to note that the total scan time is the same for all techniques. However, since these techniques have different TRs, the number of measurements is not the same for these measurements. To account for the differences in TR, we also calculated SNR and CNR of a single measurement for the techniques. Our calculations indicate that AVAST outperformed the standard ASL in terms of CNR and SNR, both in single measurement and the whole scan. Due to significantly better temporal resolution of AVAST
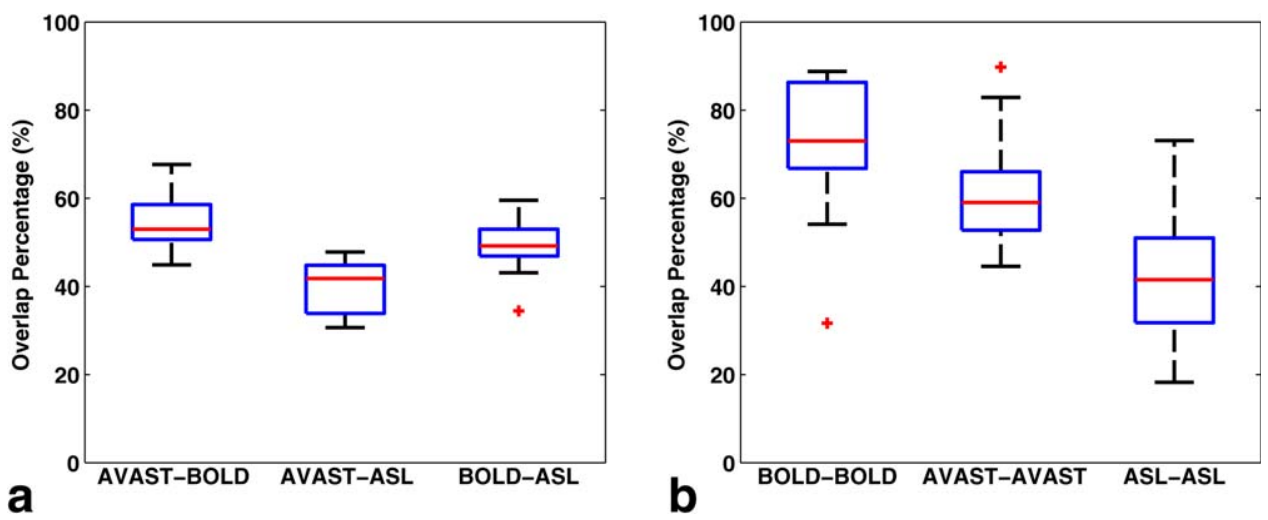

FIG. 8. Number of overlapping activated voxels detected using the AVAST, standard ASL, and BOLD methods (a) between two runs of different methods and (b) between two runs of the same method. 
Table 1

Estimated SNR and CNR Averaged Over All Subjects for Each Method

\begin{tabular}{lccc}
\hline & \multicolumn{3}{c}{ Standard } \\
& AVAST & ASL & BOLD \\
\hline CNR in activated area & 1.7 & 0.9 & 3.2 \\
SNR in whole brain & 12.9 & 6.6 & 1594 \\
CNR of single measurement & 0.026 & 0.024 & 0.021 \\
SNR of single measurement & 0.195 & 0.178 & 10.626 \\
\hline
\end{tabular}

For AVAST and standard ASL, one pair of tagged and control images was considered a single measurement.

compared with ASL, this difference is more pronounced in the whole scan. BOLD, however, provides the best CNR and SNR among all techniques. Note that the subtraction of control and tag images in AVAST and ASL experiments led to much smaller signal values compared with BOLD signals that caused a significant lower SNR for AVAST and ASL.

\section{aCBV Quantification}

Figure 9a shows an example of aCBV maps estimated in the resting state using AVAST. The aCBV values over the brain had a mean \pm standard deviation of $1.1 \pm 1$ $\mathrm{mL} / 100 \mathrm{~mL}$. Figure $9 \mathrm{~b}$ shows the histogram of the obtained values in gray matter. The large spread in gray matter aCBV values is likely related to different partial volume effects with vessels, white matter, and cerebrospinal fluid.

\section{DISCUSSION}

Inspecting both simulated and experimental kinetic curves (Fig. 2), one may notice that there are two other zero crossing points ( $\sim 0.7$ and $\sim 1.1 \mathrm{~s}$ in Fig. 2$)$ that can potentially be used for obtaining an aCBV-weighted signal. Although shorter TRs of these two points are desirable, rapid changes in total ASL around these two points would make them sensitive to transit timing variations. For that reason, the third point was used in this study. In this regime, the transit time variations led to only a small contamination from the tissue signal, since the rate of change in the ASL signal with respect to transit time is much slower.

To attain an aCBV-weighted signal with minimum contribution from the BOLD effect, the shortest TE possible is used in AVAST. While at the TE of choice in our experiment the sensitivity of the BOLD signal is approximately $<10 \%$ of that of the typical BOLD experiments (49), it may still contribute to the error of the measurement. If this contribution is severe, one can include the expected BOLD response in the general linear model as a nuisance regressor, as in $(11,50)$, and eliminate any possible BOLD contribution from the analysis. In this study, however, we have chosen to keep preprocessing to a minimum in order to show a fair comparison among techniques.

The model for ASL signal used in this study is a wellestablished model that frequently has been used and tested in the literature (33). Although more complicated models for ASL signal have been proposed in the literature $(35,43,51)$, the consistency of our experimental results with the simulation results indicate that the complexity of the model we used for our simulation is appropriate for this application.

AVAST requires a calibration scan before the fMRI scan. Once the aCBV parameters are found, however, AVAST does not require multiple measurements during the actual fMRI experiment, which is a critical advantage of this method for fMRI applications compared with other ASL-based techniques proposed for aCBV estimation (18-20,22-24). One drawback of estimating the aCBV point by finding the zero-crossing point is the lower SNR of measurements around the zero-crossing points. We circumvented this problem by averaging eight tag/control images for each TR. Another solution could be to collect a larger range of TRs on the calibration

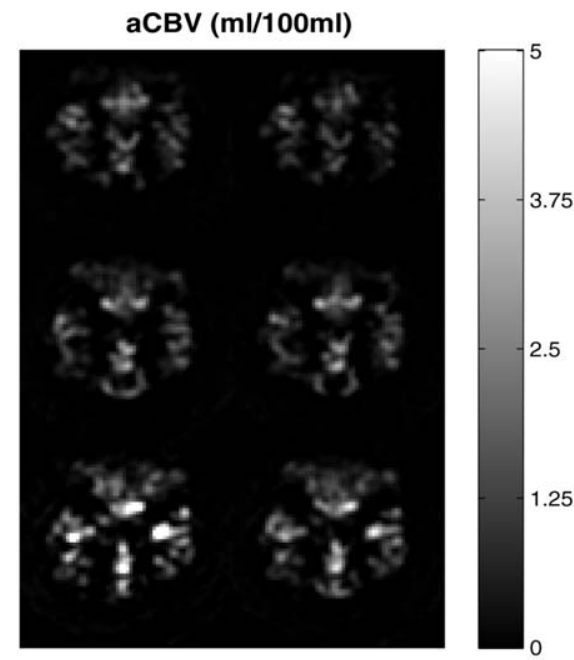

a

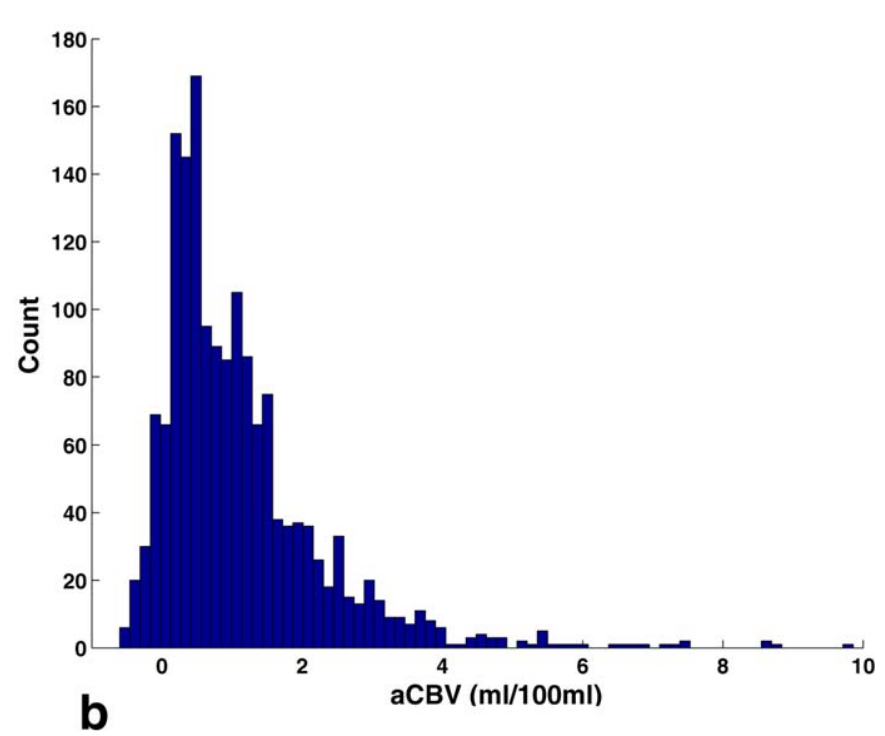

b

FIG. 9. Representative aCBV map estimated using (a) AVAST and (b) its corresponding histogram of the estimated aCBV values in gray matter. 
curve and estimating the aCBV point from a fit of the above kinetic model.

A new variant of VASO (52) dubbed iVASO has been proposed recently in order to isolate the arterial component of CBV (21). By inverting the spins in a region outside the volume of interest and collecting the images at the time that the arterial blood's magnetization is at its null point, iVASO limits the blood nulling to the incoming blood spins, which leads to an increase in SNR and CNR compared with VASO. AVAST and iVASO pulse sequences are very similar in terms of tagging the inflowing blood. The differences are that AVAST uses continuous tagging, whereas iVASO uses a pulsed tagging scheme. Most importantly, the contrast in iVASO is based on the nulling of arterial blood, whereas AVAST measures the amount of label in the arteries. However, the use of iVASO in its current implementation faces a number of challenges for fMRI applications, including slice coverage, cerebrospinal fluid contamination, and spatial variations in inversion efficiency and tissue/ blood T1. Some of these issues have been solved in recent improvements of the VASO technique (53) and can potentially be implemented for iVASO as well. Having said that, AVAST does not face these problems. Because AVAST tailors the aCBV parameters for each subject, it is potentially more reliable compared with iVASO. However, a thorough, empirical, side-by-side comparison is required to assess the advantages and disadvantages of these two methods fairly (though this comparison is beyond the scope of the present study).

There is great variability in the literature regarding the relative aCBV change arising from cerebral activation. Yan et al. (20) reported a $67.2 \%$ and $49.7 \%$ aCBV signal change using the FAIR and QUIPSS II techniques, respectively. Brookes et al. (19) reported a 33\% change in aCBV signal measured using LL-EPI-STAR technique. The range of values reported by Hua et al. using iVASO technique was $58 \pm 7 \%$ (21). Lee et al. (10) reported a $79 \%$ change in the aCBV signal. While these results are lower than the numbers we report in this study (85\%), they are within error of our results. Having said that, these discrepancies can be mostly explained by the transit time effects discussed earlier and, to a lesser degree, by different contributions from the capillary signals in each method. Differences in spatial resolution, species, presence or absence of anesthesia, stimulation paradigm, and age could also contribute to the discrepancy between our observations and previous works.

It should be noted that in both the present study as well as the mentioned previous works, the relative CBF and aCBV signal changes are different than resting state CBF-CBV coupling. This is to be expected, because arterial and venous vessels have been shown to have considerably different responses during neuronal activation and vascular challenges (e.g., hypercapnia challenge) $(10,54)$.

Although it has been shown that activation sites detected using aCBV-weighted signals are in general more localized in cortical layers (12), the spatial resolution of our study does not allow for investigating the exact location of the activation sites with respect to cortical layers and confirm this hypothesis.
The challenges to AVAST, as currently implemented, are its reduced slice coverage (compared with BOLD) and the need to acquire a resting-state calibration curve to determine the optimal sequence timing parameters. In addition, reduction in the local transit time can muddle quantification of aCBV signals during activation periods because it exaggerates the observed signal change as the result of less T1 decay. For example, if the total transit time is reduced from 1500 to $1300 \mathrm{~ms}$ without an increase in aCBV, the observed signal will be $\sim 10 \%$ greater. While this is detrimental for the quantification process, it benefits the statistical power of detecting active regions. This effect is similar to our previous observations in measuring CBF using Turbo-CASL technique (43). This effect introduces the need for a correction scheme for the overestimation of aCBV signal change during activation. One possible solution for this problem could be an approach similar to that used by Hernandez-Garcia et al. (43). Considering these facts, the application of AVAST at this current stage is confined to quantification of aCBV at rest, and detecting activationinduced aCBV changes qualitatively.

The reduction in the arterial arrival time during neural activation is also accompanied by an increase (10\%$20 \%$ ) in the local mean blood velocity. If this local change were to lead to a considerable change in carotid arteries at the tagging plane, it would cause a reduction in the tagging efficiency of the pCASL technique (55). In that case, this reduction in the tagging efficiency needs to be taken into account for quantification of the results. However, since the vasodilation happens locally at the area of activation, it is very unlikely that the neural activation would cause any detectable change in the blood velocity at the tagging plane. Also notable is that the tagging efficiency term in the model is multiplicative, meaning that changes in the efficiency would affect the amplitude of the curve, but would not shift the aCBV point.

It should also be noted that we employed a 3D stack of spirals acquisition scheme in order to eliminate differences in sensitivity to transit times across slices. Although it is a multishot sequence (since it applies the shots along the $K_{z}$ direction), it does not lead to a slice-timing shift. Instead, it produces minor blurring along the z-axis due to T1 effects and also due to inflow spins. We minimized the blurring effects by employing a cubic flipangle schedule from $15^{\circ}$ to $90^{\circ}$, as demonstrated by Nielsen and Hernandez-Garcia (44).

\section{CONCLUSION}

We have presented a new method called AVAST that uses a pseudocontinuous labeling scheme to provide an aCBV-weighted signal suitable for functional imaging experiments. Our initial results suggest that AVAST provides superior activation detection sensitivity and temporal resolution over the standard perfusion-weighted functional ASL methods, closer to those of the BOLD technique, while retaining the more desirable properties of ASL techniques. Namely, it yields a readily quantifiable physiological parameter, and because it is a subtraction technique, its noise is mostly white and not 
autoregressive. Furthermore, minimal echo times can be employed in this scheme, resulting in images that are less sensitive to susceptibility artifacts compared with BOLD-weighted imaging. AVAST can be particularly useful in longitudinal studies of cognitive function and studies requiring sustained periods of the condition or state of interest. This technique can also have a central role in the study of areas of the brain that lie near air spaces such as frontal and low brain regions which is quite challenging using BOLD effect. The proposed method can also be helpful in study of the pathological conditions during which the normal relation between $\mathrm{CBF}, \mathrm{CBV}$ and blood oxygenation is not valid.

\section{REFERENCES}

1. Rother J, Guckel F, Neff W, Schwartz A, Hennerici M. Assessment of regional cerebral blood volume in acute human stroke by use of single-slice dynamic susceptibility contrast-enhanced magnetic resonance imaging. Stroke 1996;27:1088-1093.

2. Derdeyn CP, Videen TO, Yundt KD, Fritsch SM, Carpenter DA, Grubb RL, Powers WJ. Variability of cerebral blood volume and oxygen extraction: stages of cerebral haemodynamic impairment revisited. Brain 2002;125:595-607.

3. Wu RH, Bruening R, Berchtenbreiter C, Weber J, Steiger HJ, Peller M, Penzkofer H, Reiser M. MRI assessment of cerebral blood volume in patients with brain infarcts. Neuroradiology 1998;40:496-502.

4. Alsop DC, Detre JA, Grossman M. Assessment of cerebral blood flow in Alzheimer's disease by spin-labeled magnetic resonance imaging. Ann Neurol 2000;47:93-100.

5. Aronen HJ, Gazit IE, Louis DN, et al. Cerebral blood volume maps of gliomas: comparison with tumor grade and histologic findings. Radiology 1994;191:41-51.

6. van Westen D, Petersen ET, Wirestam R, Siemund R, Bloch KM, Stahlberg F, Bjorkman-Burtscher IM, Knutsson L. Correlation between arterial blood volume obtained by arterial spin labelling and cerebral blood volume in intracranial tumours. MAGMA 2011;24:211-223.

7. Kavec M, Usenius JP, Tuunanen PI, Rissanen A, Kauppinen RA. Assessment of cerebral hemodynamics and oxygen extraction using dynamic susceptibility contrast and spin echo blood oxygenation level-dependent magnetic resonance imaging: applications to carotid stenosis patients. Neuroimage 2004;22:258-267.

8. Donahue MJ, Sideso E, MacIntosh BJ, Kennedy J, Handa A, Jezzard P. Absolute arterial cerebral blood volume quantification using inflow vascular-space-occupancy with dynamic subtraction magnetic resonance imaging. J Cereb Blood Flow Metab 2010;30:1329-1342.

9. Hua J, Qin Q, Pekar JJ, Zijl PCM. Measurement of absolute arterial cerebral blood volume in human brain without using a contrast agent. NMR Biomed 2011;24:1313-1325.

10. Lee SP, Duong TQ, Yang G, Iadecola C, Kim SG. Relative changes of cerebral arterial and venous blood volumes during increased cerebral blood flow: implications for BOLD fMRI. Magn Reson Med 2001;45: 791-800.

11. Mumford JA, Nichols T. Simple group fMRI modeling and inference. Neuroimage 2009;47:1469-1475.

12. Kim T, Kim SG. Cortical layer-dependent arterial blood volume changes: improved spatial specificity relative to BOLD fMRI. Neuroimage 2010;49:1340-1349.

13. Jin T, Kim S-G. Improved cortical-layer specificity of vascular space occupancy fMRI with slab inversion relative to spin-echo BOLD at 9.4 T. Neuroimage 2008;40:59-67.

14. Duong T, Yacoub E, Adriany G, Hu X, Ugurbil K, Vaughan J, Merkle H, Kim S. High-resolution, spin-echo BOLD, and CBF fMRI at 4 and 7 T. Magn Reson Med 2002;48:589-593.

15. Bandettini PA, Wong EC, Hinks RS, Tikofsky RS, Hyde JS. Time course EPI of human brain function during task activation. Magn Reson Med 1992;25:390-397.

16. Kim T, Hendrich KS, Masamoto K, Kim SG. Arterial versus total blood volume changes during neural activity-induced cerebral blood flow change: implication for BOLD fMRI. J Cereb Blood Flow Metab 2007;27:1235-1247.
17. Kim T, Kim S-G. Quantification of cerebral arterial blood volume and cerebral blood flow using MRI with modulation of tissue and vessel (MOTIVE) signals. Magn Reson Med 2005;54:333-342.

18. Petersen ET, Lim T, Golay X. Model-free arterial spin labeling quantification approach for perfusion MRI. Magn Reson Med 2006;55:219-232.

19. Brookes MJ, Morris PG, Gowland PA, Francis ST. Noninvasive measurement of arterial cerebral blood volume using Look-Locker EPI and arterial spin labeling. Magn Reson Med 2007;58:41-54.

20. Yan L, Li C, Kilroy E, Wehrli FW, Wang DJ. Quantification of arterial cerebral blood volume using multiphase-balanced SSFP-based ASL. Magn Reson Med 2012;68:130-139.

21. Hua J, Qin Q, Donahue MJ, Zhou J, Pekar JJ, van Zijl PCM. Inflowbased vascular-space-occupancy (iVASO) MRI. Magn Reson Med 2011;66:40-56.

22. Wang J, Alsop DC, Song HK, Maldjian JA, Tang K, Salvucci AE, Detre JA. Arterial transit time imaging with flow encoding arterial spin tagging (FEAST). Magn Reson Med 2003;50:599-607.

23. Barbier EL, Silva AC, Kim SG, Koretsky AP. Perfusion imaging using dynamic arterial spin labeling (DASL). Magn Reson Med 2001;45: 1021-1029.

24. Kim T, Kim S-G. Quantification of cerebral arterial blood volume using arterial spin labeling with intravoxel incoherent motionsensitive gradients. Magn Reson Med 2006;55:1047-1057.

25. Gunther M, Oshio K, Feinberg DA. Single-shot 3D imaging techniques improve arterial spin labeling perfusion measurements. Magn Reson Med 2005;54:491-498.

26. Maleki N, Dai W, Alsop DC. Optimization of background suppression for arterial spin labeling perfusion imaging. MAGMA 2012;25:127133.

27. Jung Y, Wong EC, Liu TT. Multiphase pseudocontinuous arterial spin labeling (MP-PCASL) for robust quantification of cerebral blood flow. Magn Reson Med 2010;64:799-810.

28. Jahanian H, Noll DC, Hernandez-Garcia L. Bo field inhomogeneity considerations in pseudo-continuous arterial spin labeling (pCASL): effects on tagging efficiency and correction strategy. NMR Biomed 2011;24:1202-1209.

29. Hua J, Donahue MJ, Zhao JM, Grgac K, Huang AJ, Zhou J, van Zijl PCM. Magnetization transfer enhanced vascular-space-occupancy (MT-VASO) functional MRI. Magn Reson Med 2009;61:944-951.

30. Dai W, Garcia D, de Bazelaire C, Alsop DC. Continuous flow-driven inversion for arterial spin labeling using pulsed radio frequency and gradient fields. Magn Reson Med 2008;60:1488-1497.

31. Wu WC, Fernandez-Seara M, Detre JA, Wehrli FW, Wang J. A theoretical and experimental investigation of the tagging efficiency of pseudocontinuous arterial spin labeling. Magn Reson Med 2007;58:1020-1027.

32. Smith AM, Lewis BK, Ruttimann UE, Ye FQ, Sinnwell TM, Yang Y, Duyn JH, Frank JA. Investigation of low frequency drift in fMRI signal. Neuroimage 1999;9:526-533.

33. Buxton RB, Frank LR, Wong EC, Siewert B, Warach S, Edelman RR. A general kinetic model for quantitative perfusion imaging with arterial spin labeling. Magn Reson Med 1998;40:383-396.

34. Chappell MA, MacIntosh BJ, Woolrich MW, Jezzard P, Payne SJ. Modelling dispersion in arterial spin labelling with validation from ASL dynamic angiography. In Proceedings of the 19th Annual Meeting of ISMRM, Montreal, Canada, 2011. p. 298.

35. Chappell MA, Woolrich MW, Kazan S, Jezzard P, Payne SJ, MacIntosh BJ. Modeling dispersion in arterial spin labeling: validation using dynamic angiographic measurements. Magn Reson Med 2013;69:563-570.

36. Herscovitch P, Raichle ME. What is the correct value for the brainblood partition coefficient for water? J Cereb Blood Flow Metab 1985; 5:65-69.

37. Lu H, Clingman C, Golay X, van Zijl P. Determining the longitudinal relaxation time (T1) of blood at 3.0 Tesla. Magn Reson Med 2004;52: 679-682.

38. Ethofer T, Mader I, Seeger U, Helms G, Erb M, Grodd W, Ludolph A, Klose U. Comparison of longitudinal metabolite relaxation times in different regions of the human brain at 1.5 and 3 Tesla. Magn Reson Med 2003;50:1296-1301.

39. Gonzalez-At JB, Alsop DC, Detre JA. Cerebral perfusion and arterial transit time changes during task activation determined with continuous arterial spin labeling. Magn Reson Med 2000;43:739-746.

40. Ye FQ, Mattay VS, Jezzard P, Frank JA, Weinberger DR, McLaughlin AC. Correction for vascular artifacts in cerebral blood flow values 
measured by using arterial spin tagging techniques. Magn Reson Med 1997;37:226-235.

41. Ye FQ, Pekar JJ, Jezzard P, Duyn J, Frank JA, McLaughlin AC. Perfusion imaging of the human brain at $1.5 \mathrm{~T}$ using a single-shot EPI spin tagging approach. Magn Reson Med 1996;36:217-224.

42. Hernandez-Garcia L, Lee GR, Vazquez AL, Noll DC. Fast, pseudocontinuous arterial spin labeling for functional imaging using a twocoil system. Magn Reson Med 2004;51:577-585.

43. Hernandez-Garcia L, Lee GR, Vazquez AL, Yip C-Y, Noll DC. Quantification of perfusion fMRI using a numerical model of arterial spin labeling that accounts for dynamic transit time effects. Magn Reson Med 2005;54:955-964.

44. Nielsen JF, Hernandez-Garcia L. Functional perfusion imaging using pseudocontinuous arterial spin labeling with low-flip-angle segmented 3D spiral readouts. Magn Reson Med 2013;69:382-390.

45. Vazquez AL, Lee GR, Hernandez-Garcia L, Noll DC. Application of selective saturation to image the dynamics of arterial blood flow during brain activation using magnetic resonance imaging. Magn Reson Med 2006;55:816-825.

46. Glover GH, Law CS. Spiral-in/out BOLD fMRI for increased SNR and reduced susceptibility artifacts. Magn Reson Med 2001;46: $515-522$.

47. Mumford JA, Nichols TE. Power calculation for group fMRI studies accounting for arbitrary design and temporal autocorrelation. Neuroimage 2008;39:261-268.
48. Hernandez-Garcia L, Jahanian H, Rowe DB. Quantitative analysis of arterial spin labeling FMRI data using a general linear model. Magn Reson Imaging 2010;28:919-927.

49. Chen NK, Egorova S, Guttmann CR, Panych LP. Functional MRI with variable echo time acquisition. Neuroimage 2003;20:2062-2070.

50. Liu T, Wong E. A signal processing model for arterial spin labeling functional MRI. Neuroimage 2005;24:207-215.

51. Chappell MA, MacIntosh BJ, Donahue MJ, Gunther M, Jezzard P, Woolrich MW. Separation of macrovascular signal in multi-inversion time arterial spin labelling MRI. Magn Reson Med 2010;63:1357-1365.

52. Lu H, Golay X, Pekar JJ, van Zijl PCM. Functional magnetic resonance imaging based on changes in vascular space occupancy. Magn Reson Med 2003;50:263-274.

53. Lu H, Hua J, van Zijl PC. Noninvasive functional imaging of cerebral blood volume with vascular-space-occupancy (VASO) MRI. NMR Biomed 2013;26:932-948.

54. Donahue MJ, Stevens RD, Hua J, Huang AJ, Pekar JJ, van Zijl PCM. Cerebral blood flow (CBF) and cerebral blood volume (CBV) coupling differs during neuronal and vascular tasks. In Proceedings of the 17th Annual Meeting of ISMRM, Honolulu, Hawaii, USA, 2009. p. 514.

55. Aslan S, Xu F, Wang PL, Uh J, Yezhuvath US, van Osch M, Lu H. Estimation of labeling efficiency in pseudocontinuous arterial spin labeling. Magn Reson Med 2010;63:765-771. 